\title{
Mecânica Atrial na Cardiomiopatia Hipertrófica: Discriminando Hipertrofia de Fibrose Ventricular
}

\author{
Atrial Mechanics in Hypertrophic Cardiomyopathy: Discriminating between Ventricular Hypertrophy and Fibrosis

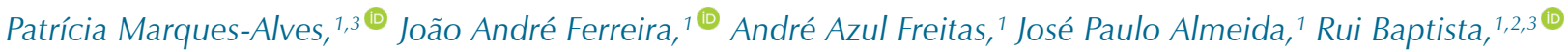 \\ Graça Castro, ${ }^{1}$ Rui Martins, ${ }^{1}$ Paulo Donato, ${ }^{3,4}$ Maria João Ferreira, ${ }^{1,2,3}$ Lino Gonçalves, ${ }^{1,2,3}$ \\ Departamento de Cardiologia, Centro Hospitalar e Universitário de Coimbra, ${ }^{1}$ Coimbra - Portugal \\ ICBR Instituto de Investigação Clínica e Biomédica de Coimbra, Universidade de Coimbra, ${ }^{2}$ Coimbra - Portugal \\ Faculdade de Medicina, Universidade de Coimbra, ${ }^{3}$ Coimbra - Portugal \\ Departamento de Radiologia, Centro Hospitalar e Universitário de Coimbra, ${ }^{4}$ Coimbra - Portugal
}

\section{Resumo}

Fundamento: A cardiomiopatia hipertrófica (CMH) e a hipertrofia ventricular esquerda (HVE) secundária à hipertensão arterial sistêmica (HAS) podem estar associadas a anormalidades funcionais do átrio esquerdo (AE).

Objetivos: Caracterizar a mecânica do AE na CMH e na HAS e avaliar qualquer correlação com a extensão da fibrose ventricular esquerda medida por ressonância magnética cardíaca (RMC) em pacientes com CMH.

Métodos: A função longitudinal do AE derivada do ecocardiograma bidimensional com speckle tracking foi adquirida a partir de cortes apicais de 60 pacientes com $\mathrm{CMH}$ e 34 indivíduos controles, pareados por idade. Pacientes com CMH também foram submetidos à RMC, com medida da extensão do realce tardio por gadolínio. A associação com parâmetros de strain do AE foi analisada. Valores $\mathrm{p}<0,05$ foram definidos como estatisticamente significativos.

Resultados: A média da fração de ejeção do ventrículo esquerdo não foi diferente entre os grupos. A razão E/e' estava comprometida no grupo $\mathrm{CMH}$ e preservada no grupo controle. A mecânica do $\mathrm{AE}$ estava significativamente reduzida na CMH em comparação aos pacientes com HAS. O strain rate do AE nas fases de reservatório (SRrAE) e na fase contrátil (SRctAE) foram os melhores parâmetros de discriminação de CMH com uma área sob a curva (AUC) de 0,8, seguido do strain do AE na fase de reservatório (SrAE) (AUC 0,76). O SRrAE e o SRctAE apresentaram elevada especificidade (89\% e 91\%, respectivamente), e o SrAE apresentou sensibilidade de $80 \%$. Um decréscimo de $2,79 \%$ no strain rate do AE na fase de condução (SRcdAE) foi preditor de um aumento de $1 \mathrm{~cm}$ na extensão do $\mathrm{RT}$ pelo gadolínio $\left(r^{2}=0,42, \beta 2,79, p=0,027\right)$.

Conclusões: O SRrAE e o SRctAE foram os melhores fatores de discriminação de HVE secundária à CMH. O SRcdAE foi preditor do grau de fibrose ventricular esquerda avaliada por RMC. Esses achados sugerem que a mecânica do AE pode ser um potencial preditor de gravidade de doença na $\mathrm{CMH}$.

Palavras-chave: Cardiomiopatia Hipertrófica; Hipertensão; Ecocardiografia/métodos; Espectroscopia de Ressonância Magnética/métodos; Hipertrofia Ventricular Esquerda.

\footnotetext{
Abstract

Background: Hypertrophic cardiomyopathy (HCM) and left ventricular hypertrophy (LVH) secondary to systemic hypertension (HTN) may be associated with left atrial (LA) functional abnormalities.

Objectives: We aimed to characterize LA mechanics in HCM and HTN and determine any correlation with the extent of left ventricular (LV) fibrosis measured by cardiac magnetic resonance (CMR) in HCM patients.

Methods: Two-dimensional speckle tracking-derived longitudinal LA function was acquired from apical views in 60 HCM patients, 60 HTN patients, and 34 age-matched controls. HCM patients also underwent CMR, with measurement of late gadolinium enhancement (LGE) extension. Association with LA strain parameters was analyzed. Statistical significance was set at $p<0.05$.

Results: Mean LV ejection fraction was not different between the groups. The E/e' ratio was impaired in the HCM group and preserved in the control group. LA mechanics was significantly reduced in HCM, compared to the HTN group. LA strain rate in reservoir (LASRr) and in contractile (LASRct) phases were the best discriminators of HCM, with an area under the curve (AUC) of 0.8, followed by LA strain in reservoir phase (LASr) (AUC 0.76). LASRr and LASR-ct had high specificity ( $89 \%$ and $91 \%$, respectively) and LASr had sensitivity of $80 \%$. A decrease in $2.79 \%$ of LA strain rate in conduit phase (LASRcd) predicted an increase of $1 \mathrm{~cm}$ in LGE extension $\left(r^{2}=0.42, \beta 2.79, p=0.027\right)$.
}

Correspondência: Patrícia Marques-Alves •

Departamento de Cardiologia, Centro Hospitalar e Universitário de Coimbra, Praceta Mota Pinto, 3000-001 Coimbra - Portugal

Email: pat.marques.alves@gmail.com

Artigo recebido em 10/08/2020, revisado em 13/01/2021, aceito em 24/02/2021

DOI: https://doi.org/10.36660/abc.20200890 
Conclusions: LASRr and LASRct were the best discriminators for LVH secondary to HCM. LASRcd predicted the degree of LV fibrosis assessed by CMR. These findings suggest that LA mechanics is a potential predictor of disease severity in HCM.

Keywords: Cardiomyopathy, Hypertrophic; Hypertension; Echocardiography/methods; Magnetic Resonance Spectroscopy/methods; Left Ventricular Hypertrophy.

Full texts in English - http://www.arquivosonline.com.br

\section{Introdução}

A hipertrofia ventricular esquerda (HVE), presente na cardiomiopatia hipertrófica $(\mathrm{CMH})$ e na hipertensão arterial sistêmica (HAS), está geralmente relacionada à disfunção do miocárdio e risco aumentado de morte súbita. ${ }^{1,2} \mathrm{Na}$ HAS, a HVE ocorre como uma resposta à sobrecarga de pressão e, na $\mathrm{CMH}$, um complexo processo de remodelamento é iniciado como uma resposta de componentes de cardiomiócitos e não-cardiomiócitos a estímulos mecânicos dinâmicos e neurohumorais..$^{1-3} \mathrm{~A} \mathrm{CMH}$ é um distúrbio autossômico dominante, associado a mutações em genes sarcoméricos, que afeta o miocárdio atrial e ventricular. ${ }^{1,2}$

O exame de ressonância magnética cardíaca (RMC) permite uma descrição abrangente de HVE e fibrose relacionadas à $\mathrm{CMH}$, por meio da técnica de realce tardio (RT) pelo gadolínio. ${ }^{4}$ Esse método, usado na avaliação quantitativa do ventrículo esquerdo (VE), permite a caracterização dos estágios da $\mathrm{CMH}$, do remodelamento e da disfunção sistólica do VE, e é um importante preditor de morte súbita., ${ }^{4,5}$ A disfunção sistólica ocorre comumente em estágios terminais da $\mathrm{CMH}$, e uma parcela significativa dos pacientes apresentam algum grau de disfunção diastólica. ${ }^{2,6}$

Aumento da massa ventricular esquerda e disfunção diastólica estão associados a dilatação e disfunção progressivas do átrio esquerdo (AE). Além disso, o remodelamento do $\mathrm{AE}$ é uma característica comum tanto da $\mathrm{CMH}$ como da HAS. ${ }^{2,7}$ Ainda, o tamanho e o volume do AE mostraram-se fatores determinantes da capacidade no exercício ${ }^{8}$ e de eventos adversos maiores cardíacos e cerebrovasculares em pacientes com $\mathrm{CMH} .{ }^{9}$

Uma vez que o AE está relacionado com o desempenho do VE dadas as suas funções de reservatório durante a sístole ventricular, condução durante a diástole ventricular precoce, e bombeamento durante a diástole ventricular tardia, a miopatia do AE poderia estar associada a desfechos independentemente da função do VE. ${ }^{10} \mathrm{~A}$ função do VE correlaciona-se com sintomas da insuficiência cardíaca na $\mathrm{CMH}$ e é forte preditora do desenvolvimento de fibrilação atrial (FA). ${ }^{11,12}$

A avaliação da mecânica do AE por meio do ecocardiograma bidimensional com speckle tracking (2D-STE) tem se mostrado um marcador viável e reprodutível para a função do AE. ${ }^{13,14}$

Apesar de a HVE parecer ser o principal fator de disfunção na mecânica do $V E,{ }^{2}$ o grau de disfunção do $\mathrm{AE}$ em diferentes estados de HVE (em particular, HVE secundária à HAS e à $\mathrm{CMH}$ ) não é totalmente entendido. Tanto a hipertrofia como a fibrose ventricular esquerda representam substratos da disfunção diastólica, e podem estar associadas à disfunção do VE na CMH. Assim, o presente estudo teve como objetivo (1) caracterizar os mecanismos do AE em pacientes com $\mathrm{CMH}$ e HAS com HVE significativa e (2) correlacionar a função do AE com fibrose do VE avaliada por RMC em pacientes com $\mathrm{CMH}$.

\section{Métodos}

\section{População do estudo}

Este estudo observacional retrospectivo incluiu 60 pacientes diagnosticados com $\mathrm{CMH}$ (diagnóstico confirmado por RMC) e 60 pacientes hipertensos recrutados no ambulatório de nosso departamento. Excluímos pacientes com CMH obstrutiva, com janela acústica inadequada, FA identificada no eletrocardiograma basal, doença valvular grave ou moderada, doença cardíaca isquêmica, ou hipertensão pulmonar definida como pressão sistólica da artéria pulmonar (PSAP) $>45 \mathrm{mmHg}$. Os pacientes foram diagnosticados com HAS cerca de 4,2 $\pm 2,3$ anos antes. Como controle, incluímos 34 pacientes sadios sem HAS, FA ou doença valvular, pareados por idade com pacientes com $\mathrm{CMH}$ e HAS.

\section{Procedimentos do estudo}

Nós analisamos dados clínicos e ecocardiográficos de participantes divididos nos grupos $\mathrm{CMH}$, HAS e controle. Dados de RMC dos pacientes com CMH no momento do diagnóstico também foram avaliados. Imagens ecocardiográficas foram coletadas $43 \pm 18$ dias após o diagnóstico de CHM por RMC. O estudo foi aprovado pelo comitê científico e pelo comitê de bioética do Centro Hospitalar e Universitário de Coimbra (Coimbra, Portugal), e foi conduzido de acordo com a Declaração de Helsinki.

\section{Dados ecocardiográficos}

Foi realizado um estudo bidimensional completo em todos os participantes, incluindo 2D-STE do VE e do AE com análise do strain longitudinal global (SLG). Utilizamos um aparelho de ultrassom cardiovascular Vivid 7 (GE Healthcare, Horten, Noruega) com um transdutor de imagem harmônica tecidual. Cortes ecocardiográficos padrões foram obtidos com otimização da taxa de quadros (60-80 fps em imagens 2D). Realizamos uma análise offline dos dados ecocardiográficos utilizando um programa específico (EchoPAC 16.0; GE Healthcare).

\section{Dimensões e função do VE}

A avaliação do tamanho e da função sistólica do VE, incluindo a medida da fração de ejeção do VE (FEVE), diâmetro diastólico final do VE (DDFVE), e diâmetro sistólico final do VE (DSFVE), seguiu as recomendações atuais. ${ }^{15} \mathrm{O}$ 


\section{Artigo Original}

SLG do VE, derivado do EST, foi obtido usando um modelo de segmentação (em 16 segmentos) do VE. ${ }^{16}$ Foi também avaliada a função diastólica, incluindo velocidade E mitral, velocidade $\mathrm{A}$ mitral, e razão E/' média.

\section{Imagens de deformação do $\mathrm{AE}$}

A análise da mecânica do AE por 2D-STE foi realizada conforme recomendado previamente, ${ }^{17,18}$ com análise offline das curvas do strain longitudinal de cada segmento atrial usando um programa específico ${ }^{17,18}$ (Figura 1). Para o processamento, o quadro (frame) inicial foi escolhido como aquele que refletisse o início da onda P. O strain e o strain rate do AE na sístole (SrAE e SRrAE, respectivamente), no início da diástole (ScdAE e SRcdAE), e no final da diástole (SctAE e SRctAE) foram medidos como indicadores das funções de reservatório, condução, e contração do $\mathrm{AE}$, respectivamente..$^{14,18}$

\section{Variabilidade entre observadores e intraobservador}

Para análise da reprodutibilidade entre observadores, as medidas de strain e strain rate por 2D-STE, obtidas de 37 pacientes com $\mathrm{CMH}$ selecionados aleatoriamente, foram realizadas por um segundo investigador (JAF) e comparadas com aquelas do primeiro observador (PMA).

O primeiro observador repetiu as medidas nos mesmos 37 participantes, e a reprodutibilidade intraobservador foi então avaliada. Os observadores avaliaram diferentes regiões de interesse do AE e desconheciam (eram cegos a) as medidas anteriores.

\section{Ressonância magnética cardíaca}

Todos os 60 pacientes com $\mathrm{CMH}$ foram submetidos à $\mathrm{RMC}$, realizada com aparelhos de 1.5 T (Philips, Best, Holanda), utilizando-se protocolos padrões, como sugerido previamente. ${ }^{4}$ As imagens de RT com gadolínio foram adquiridas 10-20 minutos após administração endovenosa do contraste, como recomendado. ${ }^{2}$ A quantificação do RT foi realizada por ajuste manual do limiar de escala de cinza, para definir áreas de RT identificadas visualmente nos planos do eixo-curto e medidas em centímetros de extensão.

\section{Análise estatística}

A normalidade da distribuição das variáveis contínuas foi avaliada por análise do histograma e o teste de Kolmogorov-
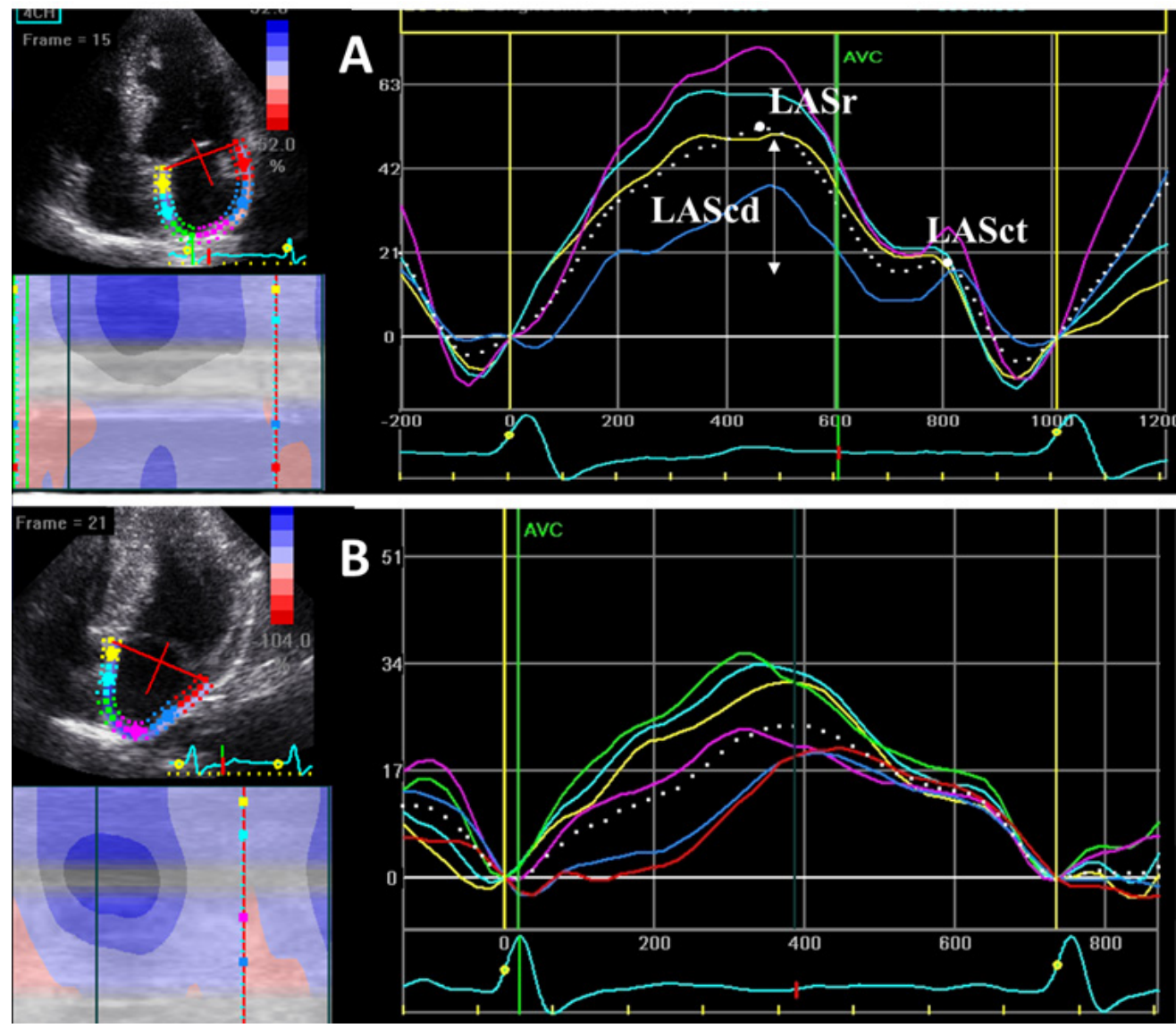

Figura 1 - Análise do strain do átrio esquerdo nos individuos controle (A) e pacientes com miocardiopatia hipertrófica (MCH) (B). Nos controles: strain do átrio esquerdo na fase de reservatório (SrAE) = 53\%; strain do átrio esquerdo na fase de contração (SctAE); ScdAE: strain do átrio esquerdo na fase de condução (diferença entre SrAE e SctAE) $=21 \%$ (A). Nos pacientes: $\operatorname{SrAE}=24 \%$; SctAE $=14 \%$ e ScdAE $=10 \%$ (B) 
Smirnov. As variáveis contínuas foram expressas em média \pm desvio padrão, e as variáveis categóricas expressas em frequência (porcentagem). Diferenças entre grupos foram avaliadas pela análise de variância (ANOVA) one-way. Após rejeitar-se a hipótese nula, o teste de comparação múltipla de Bonferroni foi realizado. Para cada variável com distribuição não normal, a homogeneidade da variância foi avaliada utilizando o teste de Levene. Para as variáveis categóricas, utilizou-se o teste do qui-quadrado ou o teste exato de Fisher, conforme apropriado. Regressão linear foi usada para correlacionar vários parâmetros contínuos. Pressupostos para a regressão linear foram verificadas antecipadamente - relação linear entre os dados foi visualmente avaliada por gráficos de dispersão; não foram detectados outliers; a autocorrelação foi excluída pelo teste de Durbin-Watson; a homogeneidade da variância dos resíduos (homoscedasticidade) também foi verificada visualmente em gráficos de resíduos versus valores ajustados. Análise de curvas da Característica de Operação do Receptor (curvas ROC) foi realizada para se estimar o poder discriminatório dos parâmetros da mecânica do $\mathrm{AE}$ na $\mathrm{CMH}$ versus $\mathrm{HA}$. As curvas foram comparadas pelo método Delong. Para a avaliação da variabilidade entre observadores e intraobservador das medidas de strain e strain rate foram utilizados método Bland-Altman, coeficiente de correlação intraclasse, e coeficiente de variação. Valores $p<0,05$ (teste bicaudal) foram considerados como estatisticamente significativos. As análises estatísticas foram realizadas utilizando o programa Stata IC para Windows (versão 13; StataCorp, Lakeway Drive, TX, EUA). Em relação ao tamanho amostral, planejamos um estudo de uma variável de resposta contínua em pacientes e controles independentes, na proporção de 0,5 indivíduos controle por paciente. $\mathrm{Na}$ análise da mecânica do AE (strain do na fase de reservatório e na fase de contração), a resposta desejável em cada grupo é normalmente distribuída com um desvio padrão de cinco. $^{7,10}$ Se a diferença entre pacientes e controle fosse quatro, necessitaríamos de 51 pacientes e 26 controles para conseguirmos rejeitar a hipótese nula de que as médias populacionais dos grupos experimental e controle fossem iguais com uma probabilidade (poder) de 0,9. A probabilidade de se cometer um erro do tipo I associado com esse teste com essa hipótese nula é de 0,05. Poderíamos ainda incluir 60 pacientes com $\mathrm{CMH}$ e 60 pacientes com HAS para melhorar o poder estatístico do estudo.

\section{Resultados}

\section{População do estudo}

As características clínicas da população do estudo estão resumidas na Tabela 1 . A idade média dos pacientes com $\mathrm{CMH}$ foi de $55 \pm 18$ anos, e $57 \%$ dos pacientes eram do sexo masculino. Esse dado não foi significativamente diferente do grupo de pacientes com HAS e controles. Os pacientes com HAS apresentaram mais diabetes mellitus, dislipidemia e obesidade.

No grupo com CHM, observou-se um uso maior de betabloqueadores, enquanto no grupo com HAS, observouse uma maior prescrição de inibidores da enzima conversora de angiotensina (IECA) e bloqueadores de receptores da angiotensina (BRA).

\section{Parâmetros ecocardiográficos convencionais}

As características ecocardiográficas dos participantes estão resumidas na Tabela 2. A média da FEVE não foi diferente entre os grupos. O DFVE foi maior no grupo com HAS que no grupo com $\mathrm{CMH}$. A PSAP não foi diferente entre os grupos $\mathrm{CHM}$ e HAS, e estava significativamente reduzida no grupo controle.

Tabela 1 - Características clínicas e demográficas da população estudada

\begin{tabular}{|c|c|c|c|c|}
\hline Característica & Controles $(n=34)$ & Grupo CMH $(n=60)$ & Grupo HAS $(n=60)$ & Valor-p \\
\hline Idade, anos ( $\pm S D)$ & $56 \pm 10$ & $55 \pm 18$ & $61 \pm 12$ & $0,081^{*}$ \\
\hline Sexo masculino (\%) & $20(55)$ & $34(57)$ & $34(57)$ & $0,124^{\#}$ \\
\hline Diabetes mellitus (\%) & 0 & $15(25)$ & $35(58)$ & $0,022^{\#}$ \\
\hline Dislipidemia (\%) & 0 & $24(40)$ & $43(72)$ & $0,014^{\#}$ \\
\hline \multicolumn{5}{|c|}{ Medicamentos anti-hipertensivos } \\
\hline Diuréticos (\%) & 0 & $15(25)$ & $32(53)$ & $0,018^{\#}$ \\
\hline Betabloqueadores & 0 & $45(75)$ & $37(62)$ & $0,068^{\#}$ \\
\hline IECA/BRA & 0 & $41(68)$ & $45(75)$ & $0,072^{\#}$ \\
\hline Outros & 0 & $12(20)$ & $20(34)$ & $0,056^{\#}$ \\
\hline
\end{tabular}

Dados apresentados como média \pm desvio padrão ou frequência (porcentagem). IECA: inibidores da enzima conversora de angiotensina; BRA: bloqueadores de receptores da angiotensina; BCC: bloqueadores de canais de cálcio; CMH: cardiomiopatia hipertrófica; HAS: hipertensão arterial sistêmica. * ANOVA one-way. \# Teste do qui-quadrado. 


\begin{tabular}{|c|c|c|c|c|c|c|}
\hline Parâmetros & Controles & Grupo HAS & Grupo CMH & p-valor global & $\begin{array}{c}\text { p-valor: } \\
\text { controles vs HAS }\end{array}$ & $\begin{array}{c}\text { p-valor: } \\
\text { CMH vs HAS }\end{array}$ \\
\hline FEVE, \% & $62,9 \pm 4,3$ & $62,9 \pm 4,9$ & $66,5 \pm 10,1$ & 0,083 & 0,969 & 0,055 \\
\hline DDFVE, $\mathrm{mm}$ & $48,3 \pm 5,2$ & $51,9 \pm 0,8$ & $49,4 \pm 1,0$ & 0,108 & 0,019 & 0,083 \\
\hline DSFVE, mm & $30,3 \pm 3,2$ & $32,3 \pm 0,7$ & $30,7 \pm 0,9$ & 0,369 & 0,119 & 0,225 \\
\hline SIV, mm & $10,2 \pm 2,8$ & $14,3 \pm 3,6$ & $16,5 \pm 5,4$ & 0,028 & $<0,001$ & 0,032 \\
\hline SLG-VE, \% & $-20,6 \pm 1,1$ & $-17,5 \pm 0,7$ & $-12,7 \pm 0,5$ & $<0,001$ & 0,192 & 0,008 \\
\hline PSAP, mmHg & $22,1 \pm 4,7$ & $26,3 \pm 0,2$ & $28,6 \pm 1,3$ & 0,021 & 0,009 & 0,245 \\
\hline VAEi, $\mathrm{mL} / \mathrm{m}^{2}$ & $23,5 \pm 4,2$ & $31,1 \pm 1,3$ & $33,5 \pm 2,5$ & $<0,001$ & 0,001 & 0,067 \\
\hline Velocidade $\mathrm{E}$ mitral, $\mathrm{m} / \mathrm{s}$ & $0,8 \pm 0,1$ & $0,7 \pm 0,2$ & $0,8 \pm 0,2$ & 0,156 & 0,068 & 0,182 \\
\hline Velocidade A mitral, m/s & $0,5 \pm 0,1$ & $0,8 \pm 0,2$ & $0,7 \pm 0,3$ & 0,005 & $<0,001$ & 0,151 \\
\hline Razão E/e' & $7,0 \pm 1,65$ & $13,2 \pm 1,2$ & $16 \pm 1,0$ & $<0,001$ & $<0,001$ & 0,035 \\
\hline SrAE, \% & $36,9 \pm 10,8$ & $24,4 \pm 8,2$ & $17,2 \pm 9,0$ & $<0,001$ & $<0,001$ & $<0,001$ \\
\hline ScdAE, $\%$ & $25,9 \pm 13,3$ & $19,9 \pm 8,7$ & $15,4 \pm 9,1$ & $<0,001$ & 0,067 & 0,022 \\
\hline SctAE, \% & $10,9 \pm 6,2$ & $5,1 \pm 0,9$ & $1,9 \pm 0,3$ & $<0,001$ & 0,003 & $<0,001$ \\
\hline SRrAE, $\%$ & $1,9 \pm 0,5$ & $1,2 \pm 0,1$ & $0,8 \pm 0,1$ & $<0,001$ & $<0,001$ & $<0,001$ \\
\hline SRcdAE, \% & $-2,1 \pm 0,6$ & $-1,8 \pm 0,1$ & $-0,6 \pm 0,1$ & $<0,001$ & 0,082 & $<0,001$ \\
\hline SRctAE, \% & $-1,9 \pm 0,7$ & $-1,7 \pm 0,1$ & $-0,9 \pm 0,1$ & $<0,001$ & 0,344 & $<0,001$ \\
\hline
\end{tabular}

Análise estatistica: one-way ANOVA com teste de Bonferroni para comparações múltiplas; CMH: cardiomiopatia hipertrófica; HAS: hipertensão arterial sistêmica; SIV: septo interventricular; SrAE: strain do átrio esquerdo na sistole (função de reservatório); ScdAE: strain do átrio esquerdo na diástole precoce (função de condução); SctAE: strain do átrio esquerdo na diástole tardia (função de contração); VAEi: volume do átrio esquerdo indexado; DDFVE: diâmetro diastólico final do ventrículo esquerdo; DSFVE: diâmetro sistólico final do ventrículo esquerdo; FEVE: fração de ejeção do ventrículo esquerdo; SLG-VE: strain longitudinal global; PSAP: pressão sistólica da artéria pulmonar.

Em relação à função diastólica do VE, a velocidade mitral E não variou entre os grupos, e a velocidade $A$ foi maior no grupo HAS. Valores anormais da razão E/e' foram encontrados no grupo com $\mathrm{CMH}$ e, no grupo controle, essa razão estava preservada.

\section{Função do AE}

Em comparação aos controles, os pacientes com $\mathrm{CHM}$ e HAS apresentaram valores de volume do AE indexado à área da superfície corporal (VAEi) significativamente maiores (Tabela 2). Os parâmetros de deformação do AE estavam reduzidos nos grupos HAS e $\mathrm{CMH}$ em relação ao grupo controle. No grupo de pacientes com HAS, a função de reservatório estava preservada, apesar de significativamente reduzida em comparação aos controles; a fase de condução não foi diferente do grupo controle, e o strain (mas não o strain rate) na fase de contração estava alterado nos pacientes com HAS (Tabela 2, Figura 2).

Todas as fases de deformação do AE estavam significativamente reduzidas no grupo $\mathrm{CMH}$ que no grupo HAS (Tabela 2, Figura 2). De todos os parâmetros da mecânica do AE, o SRrAE e o SRctAE foram os melhores discriminantes de $\mathrm{CMH}$ (versus HAS), seguido de SrAE. O SRrAR, e o SRctAE apresentaram elevada especificidade e alto valor preditivo positivo (VPP) (Tabela 3). Apesar do baixo poder discriminatório, o ScdAE apresentou a maior especificidade (94\%) e o SctAE a maior sensibilidade (95\%) (Tabela 3).

\section{Parâmetros da RMC}

Todos os pacientes com $\mathrm{CMH}$ foram submetidos à $\mathrm{RMC}$ (método padrão-ouro para o diagnóstico). O volume diastólico final indexado (VDFi) médio foi $96 \pm 32 \mathrm{~mL} / \mathrm{m}^{2}$, a espessura média do septo interventricular (SIV) foi 18,7 $\pm 3,5 \mathrm{~mm}$. Aproximadamente $34 \%$ dos pacientes apresentaram movimento sistólico anterior da valva mitral, e $12 \%$ dos pacientes apresentaram $\mathrm{CMH}$ apical. RT com gadolínio foi observado em 52 (87\%) dos pacientes com $\mathrm{CHM}$, e a média da área de extensão foi de $2,8 \mathrm{~cm}$.

\section{Parâmetros de RMC e ecocardiograma}

A Tabela 4 resume os resultados da análise de regressão linear entre a extensão do RT com gadolínio $(\mathrm{cm})$ e vários parâmetros da RMC e do ecocardiograma em pacientes com $\mathrm{CMH}$. As medidas do VE, VDFi (por RMC), DDFVE e DSFVE por ecocardiograma, o SLG do VE ou a razão E/E' não foram preditores da extensão de RT. A espessura do SIV, medida por RMC, mas não por ecocardiografia, foi preditora do RT pelo gadolínio. Tanto o SrAE como o SRcdAE foram preditores da extensão de RT pelo gadolínio. Uma redução de $0,5 \%$ no SrAE e de $2,79 \%$ no SRcdAE foram preditores de um aumento de $1 \mathrm{~cm}$ no RT pelo gadolínio (Figura 3). 
Artigo Original

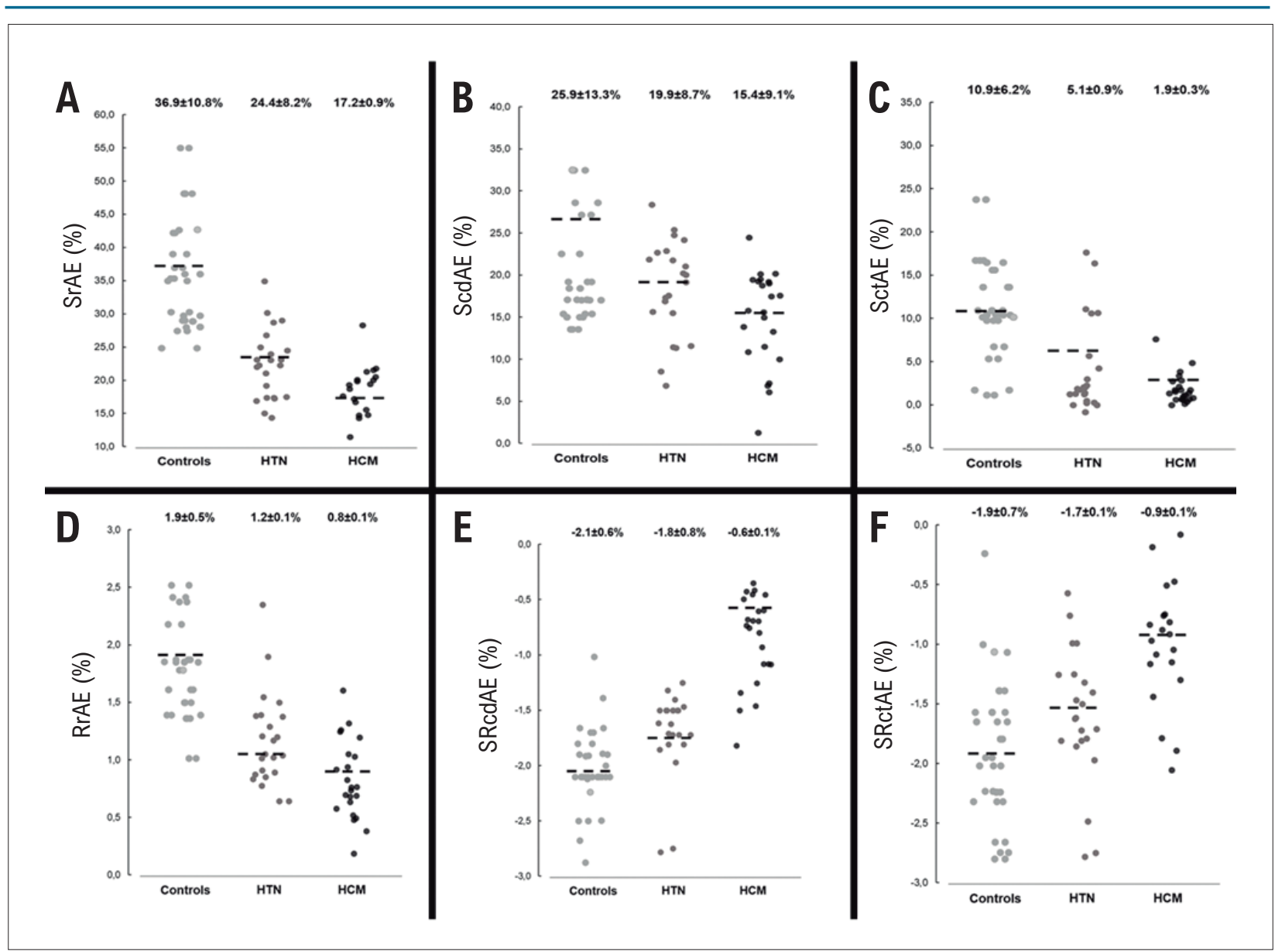

Figura 2- Parâmetros de deformação do átrio esquerdo nos pacientes com cardiomiopatia hipertrófica (CMH), pacientes com hipertensão arterial sistêmica (HAS) e controles. Strain $(A)$ e strain rate $(D)$ na fase de reservatório $(A)$; strain $(B)$ e strain rate $(E)$ na fase de condução; e strain $(C)$ e strain rate $(E)$ na fase de contração; SrAE: strain do átrio esquerdo na sistole (função de reservatório); ScdAE: strain do átrio esquerdo na diástole precoce (função de condução); SctAE: strain do átrio esquerdo na diástole tardia (função de contração); SRrAE: strain rate do átrio esquerdo na sístole (função de reservatório); SRcdAE: strain rate do átrio esquerdo na diástole precoce (função de condução); SRctAE: strain rate do átrio esquerdo na diástole tardia (função de contração).

Tabela 3 - Poder discriminatório dos parâmetros ecocardiográficos obtidos de pacientes com cardiomiopatia hipertrófica e pacientes com hipertensão arterial sistêmica

\begin{tabular}{|c|c|c|c|c|c|c|c|c|}
\hline & AUC & IC95\% & Valor $p$ & Sensibilidade (\%) & Especificidade (\%) & Critérios & VPP (\%) & VPN (\%) \\
\hline SrAE, $\%$ & 0.76 & $0.66-0.84$ & $<0.001$ & 80 & 71 & 21.8 & 73 & 78 \\
\hline ScdAE, $\%$ & 0.65 & $0.54-0.74$ & 0.012 & 32 & 94 & 9.9 & 84 & 58 \\
\hline SctAE, $\%$ & 0.65 & $0.54-0.75$ & 0.016 & 95 & 34 & 5.1 & 59 & 87 \\
\hline SRrAE, $\%$ & 0.80 & $0.71-0.88$ & $<0.001$ & 65 & 89 & 0.8 & 86 & 72 \\
\hline SRcdAE, \% & 0.69 & $0.59-0.79$ & $<0.001$ & 54 & 87 & -0.8 & 81 & 65 \\
\hline SRctAE, $\%$ & 0.80 & $0.71-0.88$ & $<0.001$ & 64 & 91 & -0.9 & 88 & 72 \\
\hline SIV (mm) & 0.62 & $0.51-0.70$ & 0.012 & 55 & 74 & 15.2 & 68 & 63 \\
\hline SLG-VE (\%) & 0.74 & $0.64-0.83$ & $<0.001$ & 57 & 84 & -13.5 & 78 & 66 \\
\hline Razão E/e' & 0.67 & $0.55-0.78$ & 0.009 & 67 & 71 & 13 & 70 & 68 \\
\hline
\end{tabular}

AUC: área sob a curva; HAS: hipertensão arterial sistêmica; CMH: cardiomiopatia hipertrófica; SIV: septo interventricular; SrAE: strain do átrio esquerdo na sístole (função de reservatório); ScdAE: strain do átrio esquerdo na diástole precoce (função de condução); SctAE: strain do átrio esquerdo na diástole tardia (função de contração); SLG-VE: strain longitudinal global; VPN: valor preditivo negativo; VPP: valor preditivo positivo. 
Tabela 4 - Análise de regressão linear entre a extensão do realce tardio por gadolínio $(\mathrm{cm})$ e parâmetros da ressonância magnética cardíaca (RMC) e de ecocardiografia

\begin{tabular}{|c|c|c|c|}
\hline Realce tardio por gadolíno & $\operatorname{Adj} \mathbf{R}^{2}$ & $\beta$ & Valor $p$ \\
\hline SIV por RMC & 0,32 & 0,12 & 0,051 \\
\hline SIV por ecocardiografia & 0,24 & 0,08 & 0,088 \\
\hline VDFi por RMC & 0,01 & 0,01 & 0,843 \\
\hline DDFVE & 0,01 & $-0,02$ & 0,795 \\
\hline Razão E/E' & 0,01 & $-0,04$ & 0,802 \\
\hline SLG-VE & 0,04 & 0,08 & 0,467 \\
\hline SrAE & 0,12 & $-0,02$ & 0,085 \\
\hline ScdAE & 0,15 & $-0,01$ & 0,092 \\
\hline SctAE & 0,35 & $-0,51$ & 0,045 \\
\hline SRrAE & 0,12 & $-1,29$ & 0,073 \\
\hline SRcdAE & 0,42 & 2,79 & 0,027 \\
\hline SRctAE & 0,21 & 0,33 & 0,066 \\
\hline
\end{tabular}

RMC: ressonância magnética cardíaca; VDFi: volume diastólico final indexado; SIV: septo interventricular; SrAE: strain do átrio esquerdo na sistole (função de reservatório); ScdAE: strain do átrio esquerdo na diástole precoce (função de condução); SctAE: strain do átrio esquerdo na diástole tardia (função de contração); SLG-VE: strain longitudinal global; DDFVE: diâmetro diastólico final do ventrículo esquerdo.

Variabilidade entre observadores e intraobservador nas medidas de deformação do AE por 2D-STE

Os parâmetros de deformação do AE mostraram valores de coeficiente de correlação intraclasse de 0,64 a 0,94, indicando uma boa a excelente reprodutibilidade dessas medidas (Tabela suplementar). Os gráficos de Bland-Altman revelaram uma discrepância muito baixa entre observadores (Figura 4) e intraobservadores (Figura 5) quanto às medidas de strain a strain rate do $\mathrm{AE}$.

\section{Discussão}

O presente estudo avaliou a mecânica do AE na HVE secundária à HAS e $\mathrm{CMH}$, e analisou se o remodelamento do AE esteve associado com a extensão de HVE e fibrose na $\mathrm{CMH}$. Os resultados fornecem importantes informações acerca da função do $\mathrm{AE}$, conforme identificado em um estudo preliminar. ${ }^{19}$ Conseguimos demonstrar que a mecânica do $\mathrm{AE}$ está diminuída tanto na HAS como na $\mathrm{CMH}$. A função do $\mathrm{AE}$ está prejudicada na $\mathrm{CMH}$ ao se comparar com a HAS (Tabela 2, Figura 2). Os parâmetros que melhor discriminaram $\mathrm{CMH}$ foram o SRrAE e o SRctAE, com uma área sob a curva de 0,8, e um valor preditivo positivo de $86 \%$ e $88 \%$, respectivamente. O ScdAE apresentou a maior especificidade (94\%) e o SctAE a maior sensibilidade (95\%) (Tabela 3). Ainda, nós conseguimos demonstrar uma correlação moderada entre a mecânica do AE e o grau de fibrose avaliada por RMC nos pacientes com $\mathrm{CHM}$, entre o SRcdAE e a extensão de RT pelo gadolínio (Tabela 4).

O comprometimento na função de reservatório do AE é acompanhado por uma deterioração da função do VE, e é maior nos pacientes com CHM que nos pacientes com HA. A redução na função de reservatório do $\mathrm{AE}$ na $\mathrm{CHM}$ está relacionada à disfunção longitudinal do $\mathrm{VE}$, devido à diminuição da sístole na base do VE, o que leva a um comprometimento no relaxamento e consequente rigidez do $A E .^{7,20}$ De fato, foi demonstrada recentemente uma associação significativa entre a função de reservatório do AE (SrAE) e um pior desfecho nos pacientes com $\mathrm{CMH}$, com uma correlação linear entre SrAE e níveis de peptídeo natriurético tipo-B. ${ }^{21}$

Apesar de a função de condução do AE estar associada à função sistólica do VE (dissincronia ventricular), ela está relacionada à extensão de hipertrofia., ${ }^{2,20} \mathrm{Em}$ nosso estudo, $\mathrm{O}$ SRcdAE foi o parâmetro que melhor se correlacionou com a extensão da fibrose do VE. Tal fato deve-se provavelmente à redução da complacência do VE devido à fibrose do miocárdio, com redução da função de condução atrial na $\mathrm{CMH}$.

A fase contrátil do AE também esteve comprometida na $\mathrm{CMH}$, o que é de certa forma inconsistente com estudos prévios que mostraram uma tendência de aumento (embora não estatisticamente significativo) da função contrátil e de bombeamento na CMH com ausência de fibrose do VE. ${ }^{2}$ Isso pode estar relacionado ao fato de que $87 \%$ dos nossos pacientes com CMH já apresentavam RT com gadolínio na RMC e, assim, possível comprometimento da função contrátil do AE.

Quanto à presença de fibrose, um estudo mostrou uma correlação significativa entre o RT por gadolínio do AE e do VE na RMC, bem como um strain do VE anormal. ${ }^{22}$ Tal fato pode sugerir que a fibrose do AE seja secundária ao remodelamento do VE e aumento da pressão de enchimento. Apesar de a mecânica do AE não ter sido investigada por 2D-STE no presente estudo, podemos relacionar esses achados com nossos resultados sobre a mecânica do AE: comprometimento das funções de reservatório e contrátil, e uma correlação entre função de condução e extensão da fibrose do VE.

Apesar de a disfunção do VE, avaliada pelo SLG do VE, estar muito presente no grupo com $\mathrm{CMH}$, não se observou uma relação com fibrose do VE. Outro parâmetro da disfunção do VE, o índice de performance miocárdica (IPM), também está comprometido na $\mathrm{CMH}$ e está relacionado com o strain do $\mathrm{AE}$. 
A

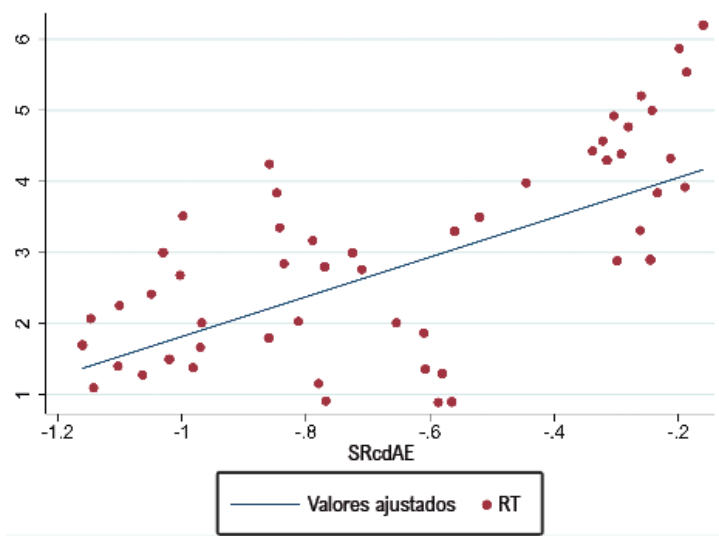

B

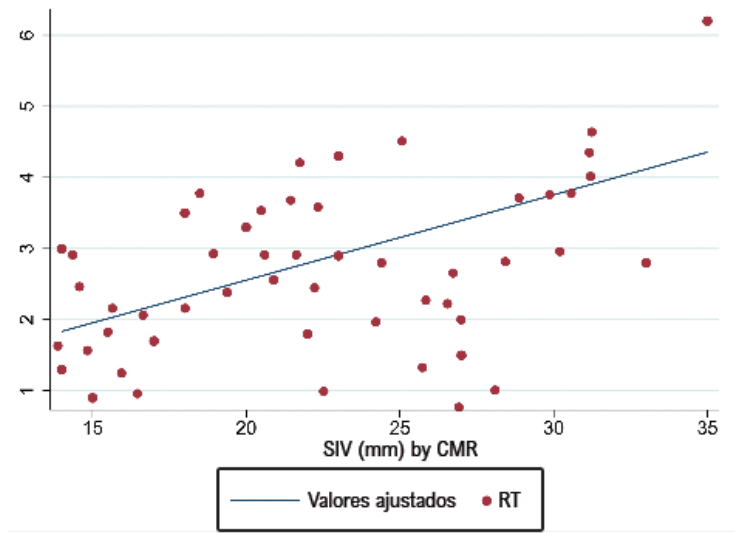

C

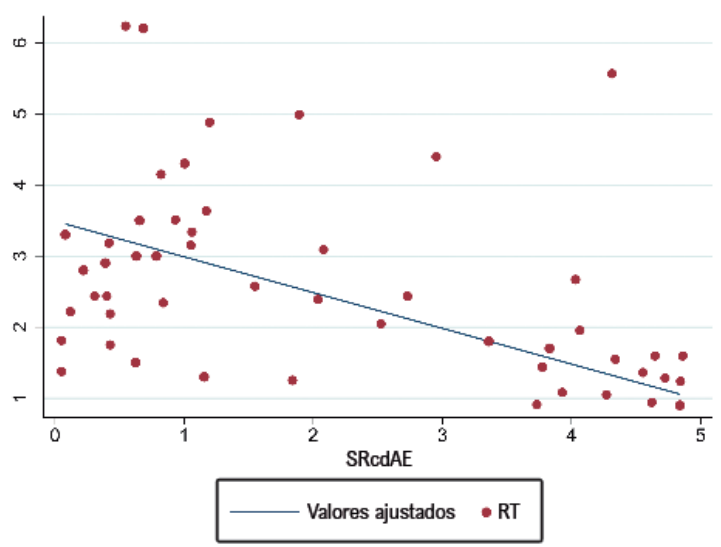

Figura 3 - Correlação linear entre extensão do realce tardio (RT) por gadolínio e espessura do septo interventricular (SIV) medida por ressonância magnética cardiaca $(R M C)(A)$, strain do átrio esquerdo na diástole tardia (função de contração) (SctAE) (B) strain rate do átrio esquerdo na diástole precoce (função de condução) (SRcdAE) (C).

Contudo, o IPM não foi preditivo do desfecho, diferentemente do strain do AE. ${ }^{23}$

Ao avaliar a disfunção diastólica na $\mathrm{CMH}$ versus HAS, observamos que o VAEi e as velocidades E e A mitral não foram diferentes entre os grupos. Tal fato demonstra a importância da mecânica do AE como um fator discriminante de miopatia do $\mathrm{AE}$, a qual está comprometida mesmo antes de uma dilatação atrial esquerda. Ainda, esse dado estabelece valores basais de deformação do $A E$, que não sejam influenciados por outros fatores tais como FA (excluída) ou dilatação atrial esquerda importante. Com a progressão da doença, podemos esperar que a deformação do $\mathrm{AE}$ piora com a dilatação do $\mathrm{AE}$, apesar de não termos provado isso em nosso estudo. Apesar dos diferentes valores da relação $E / E^{\prime}$, o parâmetro não foi um bom parâmetro de discriminação entre os grupos e não se correlacionou com a extensão da fibrose miocárdica do VE na $\mathrm{CMH}$. A mecânica do AE parece mais um fator discriminatório entre os grupos com HVE e está relacionada ao grau de fibrose miocárdica do VE na $\mathrm{CMH}$. Esses achados também foram relatados em outras cardiomiopatias, em que não se observaram diferenças nos valores de E/E' ou VAEi entres os três grupos de cardiomiopatia $\left(\mathrm{CMH}\right.$, restritiva e dilatada)..$^{24}$ Ainda, o strain e o strain rate foram melhores preditores de cardiomiopatias. ${ }^{24}$ Tal fato sugere que a mecânica do $\mathrm{AE}$ seja um marcador mais precoce tanto de disfunções atrial como miocárdica. Além disso, em pacientes com HAS e HVE significativa, nos quais a exclusão de $\mathrm{CMH}$ pode ser um desafio, a avaliação da mecânica do $\mathrm{AE}$ pode ser útil, uma vez que as três fases (de reservatório, condução e contração) não estão afetadas nesse grupo. Em nosso estudo, apesar de estatisticamente mais baixos em comparação a pacientes com $\mathrm{CMH}$, os valores da espessura do SIV na nossa coorte de pacientes hipertensos ainda estavam elevados (média de $14,3 \pm 3,6 \mathrm{~mm})$.

Em nosso estudo, demonstramos que o strain e o strain rate do AE são fatores discriminatórios potenciais entre $\mathrm{CMH}$ e HAS, não somente na resposta fisiológica à HVE como determinantes da disfunção. As implicações clínicas para o uso do strain rate do AE para o diagnóstico de CMH em pacientes com HVE ainda são incertas, uma vez que a RMC é o método de imagem mais 


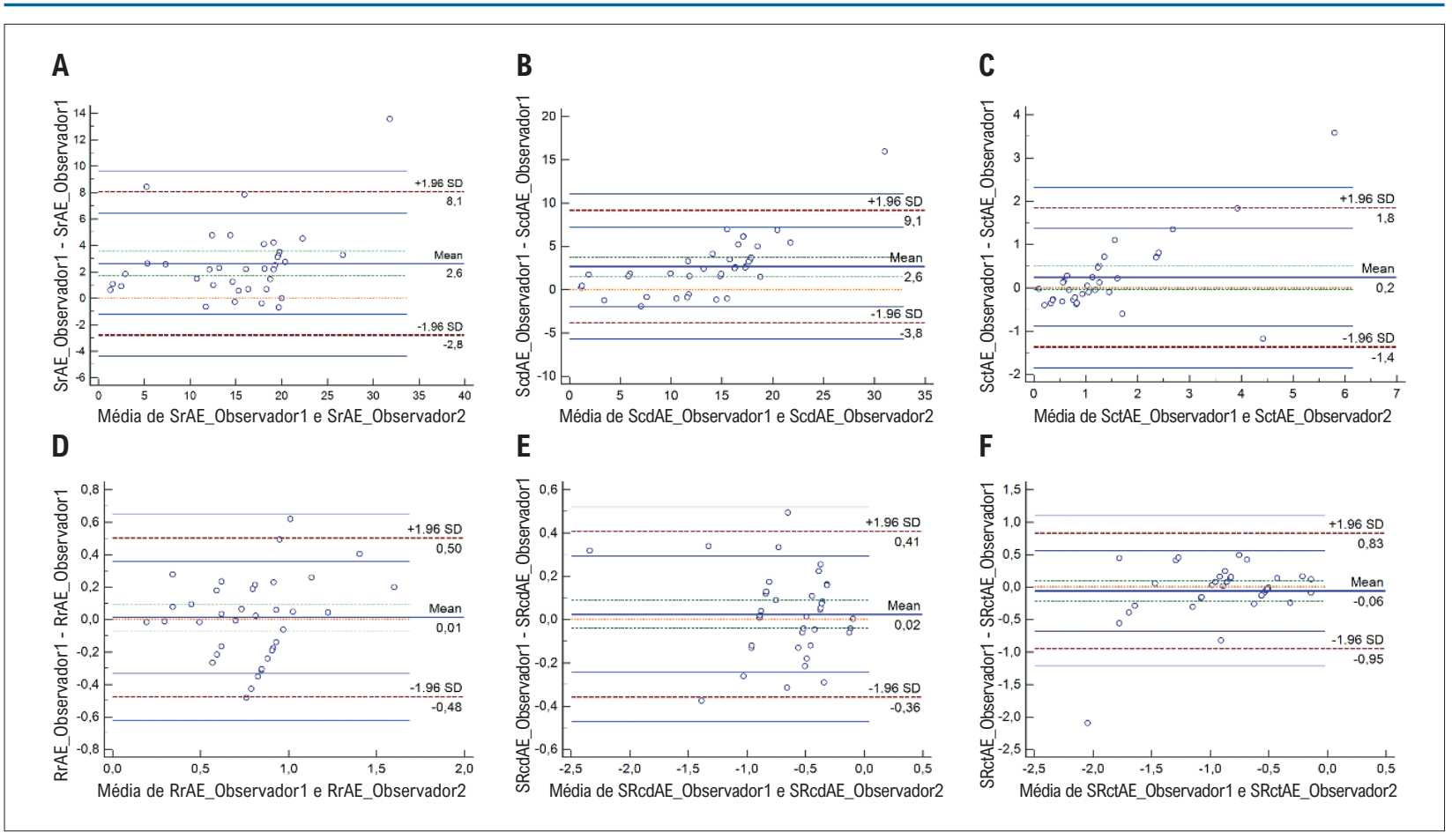

Figura 4 - Gráficos Bland-Altman plots para variabilidade entre observadores quanto às medidas de strain $(A, B, C)$ e strain rate $(D, E, F)$ do átrio esquerdo. SrAE: strain do átrio esquerdo na sistole (função de reservatório); ScdAE: strain do átrio esquerdo na diástole precoce (função de condução); SctAE: strain do átrio esquerdo na diástole tardia (função de contração); SRrAE: strain rate do átrio esquerdo na sístole (função de reservatório); SRcdAE: strain rate do átrio esquerdo na diástole precoce (função de condução); SRctAE: strain rate do átrio esquerdo na diástole tardia (função de contração).

preciso. Contudo, pudemos demonstrar que a mecânica do AE foi um fator discriminatório mais forte de HVE secundária à $\mathrm{CMH}$ quando comparada a outros parâmetros clássicos, como o VAEi, razão E/E', e mesmo espessura do SIV (Tabelas 2 e 3). Ainda, observou-se uma correlação moderada entre a mecânica do $\mathrm{AE}$ e a extensão de fibrose do $\mathrm{AE}$ na $\mathrm{CMH}$, o que poderia se tonar um marcador de gravidade e prognóstico em estágios iniciais ou casos duvidosos.

\section{Limitações}

Nosso estudo tem várias limitações. Primeiro, o programa usado para a análise de strain foi dedicado à análise do strain do $\mathrm{VE}$, e não do $\mathrm{AE}$, o que poderia distorcer de alguma forma nossos resultados.

Segundo, a heterogeneidade da população do nosso estudo, com diferentes comorbidades, pode afetar os resultados obtidos. O fato de que uma maior proporção de pacientes hipertensos apresentou diabetes e obesidade poderia afetar a análise da mecânica do $A E$, devido à piora na função diastólica do VE. No entanto, essa é uma associação intrínseca que não pôde ser excluída, uma vez que há poucos pacientes que apresentassem HAS como o único fator de risco cardiovascular. A maioria dos pacientes eram adequadamente tratados com IECA e BRA, o que poderia ter afetado a remodelação do VE e compensado a disfunção sistólica nesses pacientes. Em contraste, os betabloqueadores na $\mathrm{CMH}$ não afetam diretamente a remodelação do VE, não influenciando, assim, a função diastólica do VE ou a mecânica do AE. No entanto, nós não pudemos avaliar se, e em que extensão tal fato tenha ocorrido. Contudo, a partir de uma comparação direta de dois grupos heterogêneos de pacientes do mundo real com $\mathrm{HA}$ ou $\mathrm{CMH}$, podemos avaliar as detectar diferenças na mecânica do AE entre esses dois grupos, conforme observado na prática diária.

Terceiro, um estudo ${ }^{25}$ demonstrou que pacientes com $\mathrm{CMH}$ com FA paroxística podem apresentar um maior grau de miopatia do AE que pacientes sem FA. Em nosso estudo, apesar de termos excluído pacientes com FA no eletrocardiograma basal, é possível a existência de pacientes com FA paroxística que não tenham sido identificados, com um fenótipo cardíaco mais grave, que não foram totalmente caracterizados em nossa análise.

Quarto, nós não temos a validação externa dos pontos de corte propostos, de modo que não podemos sugerir propriamente o uso do strain do AE como um fator de discriminação preciso.

Quinto, não incluímos pacientes com HAS na análise de variabilidade, o que seria importante para avaliar realmente a reprodutibilidade de todos os parâmetros.

Finalmente, uma vez que este foi um estudo baseado em desfechos, não podemos tirar conclusões acerca do valor prognóstico do strain rate do AE nessa população. No entanto, este estudo tentou esclarecer a mecânica da deformação do $\mathrm{AE}$ na HVE secundária à $\mathrm{CMH}$ e à HAS. Mais estudos com um tamanho amostral maior são 


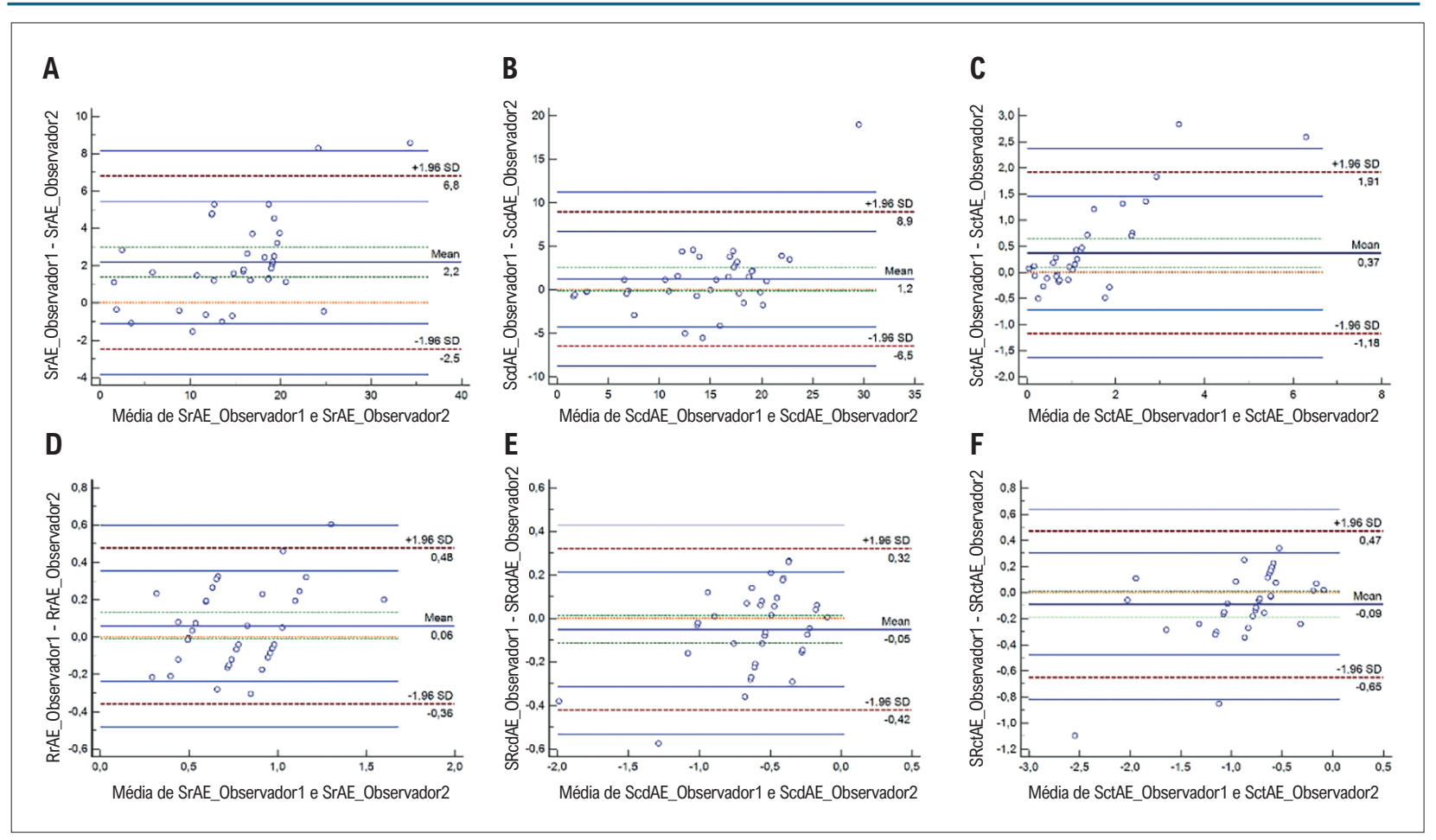

Figura 5 - Gráficos Bland-Altman plots para variabilidade intraobservador das medidas de strain $(A, B, C)$ e strain rate $(D, E, F)$ do átrio esquerdo. SrAE: strain do átrio esquerdo na sístole (função de reservatório); ScdAE: strain do átrio esquerdo na diástole precoce (função de condução); SctAE: strain do átrio esquerdo na diástole tardia (função de contração); SRrAE: strain rate do átrio esquerdo na sistole (função de reservatório); SRcdAE: strain rate do átrio esquerdo na diástole precoce (função de condução); SRctAE: strain rate do átrio esquerdo na diástole tardia (função de contração).

necessários para esclarecer o valor prognóstico do strain rate do AE na HVE.

\section{Conclusão}

A mecânica do AE está globalmente comprometida na HVE secundária à HAS e à $\mathrm{CMH}$. Em comparação à HAS, os melhores fatores de discriminação de $\mathrm{CMH}$ foram SRrAE e SRctAE. No entanto, o SRcdAE correlacionou-se melhor com o grau de fibrose do VE avaliada por RMC nos pacientes com $\mathrm{CMH}$. Esses achados sugerem que a mecânica do $\mathrm{AE}$ pode ajudar na diferenciação de HVE entre HAS e CMH e ser um preditor potencial de gravidade da doença na $\mathrm{CMH}$.

\section{Contribuição dos autores}

Concepção e desenho da pesquisa, Análise estatística e Redação do manuscrito: Marques-Alves P; Obtenção de dados:
Marques-Alves P, Ferreira JA, Freitas AA, Almeida JP; Análise e interpretação dos dados: Marques-Alves $P$, Ferreira JA, Freitas AA, Almeida JP, Baptista R; Revisão crítica do manuscrito quanto ao conteúdo intelectual importante: Marques-Alves P, Baptista R, Castro G, Martins R, Donato P, Ferreira MJ, Gonçalves L.

\section{Potencial conflito de interesse}

Não há conflito com o presente artigo

\section{Fontes de financiamento}

O presente estudo não teve fontes de financiamento externas.

\section{Vinculação acadêmica}

Não há vinculação deste estudo a programas de pósgraduação.

\section{Referências}

1. Niimura H, Patton KK, McKenna WJ, Souts J, Maron B, Seidman JG.et al. Sarcomere protein gene mutations in hypertrophic cardiomyopathy of the elderly. Circulation. 2002;105(4):446-451. doi:10.1161/hc0402.102990

2. Kowallick JT, Vieira MS, Kutty S, Lotz J, Hasenfu G, Charibin A, Schuster A. Left Atrial Performance in the Course of Relation to Left Ventricular Hypertrophy and Fibrosis. Invest Radiol 2017;52(3):177-85. doi:10.1097/RLI.0000000000000326
3. De Simone G, Pasanisi F, Contaldo F. Link of nonhemodynamic factors to hemodynamic determinants of left ventricular hypertrophy. Hypertension. 2001;38(1):13-8. doi:10.1161/01.HYP.38.1.13

4. Maron MS. Clinical utility of cardiovascular magnetic resonance in hypertrophic cardiomyopathy. J Cardiovasc Magn Reson. 2012;14(1):12-15. doi:10.1186/1532-429X-14-13 
5. Bruder O, Wagner A, Jensen CJ, et al. Myocardial scar visualized by cardiovascular magnetic resonance imaging predicts major adverse events in patients with hypertrophic cardiomyopathy. J Am Coll Cardiol. 2010;56(11):875-887. doi:10.1016/j.jacc.2010.05.007

6. Rakowski H, Carasso S. Quantifying diastolic function in hypertrophic cardiomyopathy: The ongoing search for the Holy Grail. Circulation. 2007;116(23):2662-5. doi:10.1161/CIRCULATIONAHA.107.742395

7. Vasquez N, Ostrander BT, Lu D, Ventoulis I, Haileselassie, Goyal S, et al. Low Left Atrial Strain Is Associated With Adverse Outcomes in Hypertrophic Cardiomyopathy Patients. J Am Soc Echocardiogr. 2019;32(5):593-603. e1.doi:10.1016/j.echo.2019.01.007

8. Sachdev V, Shizukuda Y, Brenneman CL, birdsall CW, Waclawiw MA, Arai $\mathrm{AE}$, et al. Left atrial volumetric remodeling is predictive of functional capacity in nonobstructive hypertrophic cardiomyopathy. Am Heart J. 2005;149(4):730-6. doi:10.1016/J.AHJ.2004.07.017

9. Nistri S, Olivotto I, Betocchi S, Losi MA, Valsecchi G, Pinamonti B, et al. Prognostic significance of left atrial size in patients with hypertrophic cardiomyopathy (from the Italian Registry for Hypertrophic Cardiomyopathy). Am J Cardiol. 2006;98(7):960-5. doi:10.1016/j. amjcard.2006.05.013

10. Hoit BD. Left atrial size and function: Role in prognosis. J Am Coll Cardiol. 2014;63(6):493-505. doi:10.1016/j.jacc.2013.10.055

11. Williams LK, Chan RH, Carasso S, Durand M, Misurka J, Crean AH, et al. Effect of Left Ventricular Outflow Tract Obstruction on Left Atrial Mechanics in Hypertrophic Cardiomyopathy. Biomed Res Int. 2015;2015:481245

12. Maron BJ, Haas TS, Maron MS, Durand M, Misuurka J, Crean AM, et al. Left atrial remodeling in hypertrophic cardiomyopathy and susceptibility markers for atrial fibrillation identified by cardiovascular magnetic resonance. Am J Cardiol. 2014;113(8):1394-400. doi:10.1016/j.amjcard.2013.12.045

13. Vieira MJ, Teixeira R, Gonçalves L, Gersh BJ. Left atrial mechanics: Echocardiographic assessment and clinical implications. J Am Soc Echocardiogr. 2014;27(5):463-478. doi:10.1016/j.echo.2014.01.021

14. Marques-Alves P, Marinho AV, Domingues C, Baptista R, Castro G, Martins R, et al. Left atrial mechanics in moderate mitral valve disease: earlier markers of damage. Int J Cardiovasc Imaging. 2019;36(1):23-31. doi:10.1007/ s10554-019-01683-w

15. Lang RM, Badano LP, Mor-Avi V, Afilalo J, Amstrong A, Ernande L, et al. Recommendations for cardiac chamber quantification by echocardiography in adults: An update from the American society of echocardiography and the European association of cardiovascular imaging. J Am Soc Echocardiogr. 2015;16(3):233-71. doi:10.1093/ehjci/jev014

16. Voigt JU, Pedrizzetti G, Lysyansky P, Marweck TH, Houle H, Baumann $\mathrm{R}$, et al. Definitions for a common standard for 2D speckle tracking echocardiography: consensus document of the EACVI/ASE/Industry Task Force to standardize deformation imaging. Eur Heart J Cardiovasc Imaging. 2015;16(1):1-11. doi:10.1093/ehjci/jeu184

17. Todaro MC, Choudhuri I, Belohlavek M, Jahangir A, Carery S, Oreto $\mathrm{L}$, et al. New echocardiographic techniques for evaluation of left atrial mechanics. Eur Heart J Cardiovasc Imaging. 2012;13(12):973-84. doi:10.1093/ehjci/jes174

18. Badano LP, Kolias TJ, Muraru D, Abraham T, Aurigemm G, Edvardsen $J$, et al. Standardization of left atrial, right ventricular, and right atrial deformation imaging using two-dimensional speckle tracking echocardiography: A consensus document of the EACVI/ASE/Industry Task Force to standardize deformation imaging. Eur Heart J Cardiovasc Imaging. 2018;19(6):591-600. doi:10.1093/ehjci/jey042

19. Alves $P$, Leite $L$, Baptista R. Left atrial mechanics through strain analysis can differentiate hypertrophic cardiomyopathy from hypertrophy secondary to arterial hypertension. Eur Heart J. 2017Aug 20;38(Suppl 1):P2417. doi:10.1093/eurheartj/ehx502.P2417

20. Badran HM, Faheem N, Elnoamany MF. Characterization of Left Atrial Mechanics in Hypertrophic Cardiomyopathy and Essential Hypertension Using Vector Velocity Imaging. 2015:1527-38. doi:10.1111/echo.12885

21. Essayagh B, Resseguier N, Michel N,Casalta AC, Renard S, Donghi V. et al. Left atrial dysfunction as marker of poor outcome in patients with hypertrophic cardiomyopathy. Arch Cardiovasc Dis. 2020; 114(2):96104. doi:10.1016/j.acvd.2020.06.004

22. Latif SR, Nguyen VQ, Peters DC, Soufer A, Henry ML, Grunseich K, et al. Left atrial fibrosis correlates with extent of left ventricular myocardial delayed enhancement and left ventricular strain in hypertrophic cardiomyopathy. Int J Cardiovasc Imaging. 2019;35(7):1309-18. doi:10.1007/s10554-019-01551-7

23. Kobayashi Y, Moneghetti KJ, Bouajila S, Clolfo D, Achley E, Wheeler M, et al. Time based versus strain basedmyocardial performance indices in hypertrophic cardiomyopathy, themerging role of left atrial strain. Eur Heart J Cardiovasc Imaging. 2019;20(3):334-42. doi:10.1093/ehjci/ jey097

24. Sabatino J, Di Salvo G, Prota C, Bucciarelli V, Josen M, Paredes J, et al. Left Atrial Strain to Identify Diastolic Dysfunction in Children with Cardiomyopathies. J Clin Med. 2019;8(8):1243. doi:10.3390/ jcm8081243

25. Sivalokanathan S, Zghaib T, Greenland G V, Vasquez N, Kudchadkar S, Kontari E, et al. Hypertrophic Cardiomyopathy Patients With Paroxysmal Atrial Fibrillation Have a High Burden of Left Atrial Fibrosis by Cardiac Magnetic Resonance Imaging. JACC Clin Electrophysiol. 2019;5(3):36475. doi:10.1016/j.jacep.2018.10.016

\author{
* Material suplementar \\ Para informação adicional, por favor, clique aqui.
}

